\title{
Time-resolved measurement of a self-amplified free-electron laser
}

\author{
Yuelin Li ${ }^{*}$, John Lewellen, Zhirong Huang, Vadim Sajaev, and Stephen V. Milton \\ Advanced Photon Source, Argonne National Laboratory, Argonne, IL 604439
}

We report on a time-resolved measurement of self-amplified spontaneous emission (SASE) free-

electron laser (FEL) pulses. We observed that the spikes in the output of such free-electron laser pulses have an intrinsic positive chirp and the energy chirp in the electron bunch mapped directly into the FEL output. The measurement also provides rich information on the statistics of the FEL output.

PACS: 41.60.-m, 41.60.Cr, 42.25.Bs, 42.55.Vc, 52.59.Rz

Keyword: Self-Amplified spontaneous emission; phase measurement; energy chirp; statistic analysis

\footnotetext{
* Corresponding author, Argonne National Laboratory, Bldg 401, Rm B2196, Argonne, IL 60439, Tel: (630) $252-$ 7863, Fax: (630) 252-5703, Email ylli@aps.anl.gov
}

The submitted manuscript has been created by the University of Chicago as Operator of

Argonne National Laboratory ("Argonne") under

Contract No. W-31-109-ENG-38 with the U.S.

Department of Energy. The U.S. Government

retains for itself, and others acting on its behalf,

a paid-up, nonexclusive, irrevocable worldwide

license in said article to reproduce, prepare

derivative works, distribute copies to the public,

and perform publicly and display publicly, by or

on behalf of the Government. 
Single-pass, high-gain free-electron lasers (FELs) based on self-amplified spontaneous emission (SASE) $[1,2]$ are proposed for the next generation of high-brightness, coherent X-ray sources with ultrashort pulse durations $[3,4]$. Recent experiments have demonstrated saturation of such SASE FELs [5-7] and their capability of achieving shorter and tunable wavelengths by direct amplification [6] as well as harmonic generations $[7,8]$.

In a SASE FEL, a favorable instability occurs due to the interaction of an electron beam and the electromagnetic wave it produces as the beam propagates down an undulator. Provided the interaction is strong enough, the radiation power grows exponentially with the undulator distance until it reaches saturation [1,2]. We report the first single-shot time-resolved characterization of SASE pulses using the frequency-resolved optical gating (FROG) technique [9]. The measurement revealed the phase of the FEL output and temporal and spectral structures and their fluctuation.

The measurements were conducted at the low-energy undulator test line at the Advanced Photon Source $[5,10]$. Table 1 is a summary of the main parameters for this experiment. Briefly, a high-brightness electron bunch generated from an rf photocathode gun is compressed through a magnetic chicane and then accelerated to $217 \mathrm{MeV}$ in energy and sent into an undulator line. A mirror at each station can also direct the SASE light toward diagnostics located outside of the tunnel.

Negative energy chirp of the electron bunch $\sigma_{\delta} / \sigma_{\mathrm{z}}$ in data set A is determined by energy spread on a spectrometer using a modified linac zero-phasing technique. Here $\sigma_{\delta}$ is the relative correlated energy spread, and $\sigma_{\mathrm{z}}$ is the rms bunch length. Exponential gain and saturation were verified by measuring the FEL output as a function of the distance. 
A single-shot frequency resolved optical gating (FROG) device using the second harmonic gating geometry [9] records single-shot spectrograms, where the recorded signal is $I_{F R O G}(\omega, \tau) \propto\left|\int_{-\infty}^{\infty} E_{s i g}(t, \tau) \exp (-i \omega t) d t\right|^{2}$ and contains both amplitude and phase of the input. It also measures the relative output energy of the FEL.

Example traces of the FROG measurement along with the retrieved pulse shape, phase, and instantaneous frequency in the time domain are given in Figs. 1 (a) and (b). Note that the instantaneous frequency is obtained by differentiating the phase, hence spikes caused by phase discontinuity should be disregarded.

In general, the traces measured by the FROG have large fluctuations. Figure 1 shows two extreme cases. Figure 1 (a) depicts a single spike-dominated trace, and Fig. 1 (b) shows a multiple spike pulse. The asymmetry in the frequency axis in both traces is an indication of overall energy chirp. While the phases for each spike are very complicated, there are clearly phase discontinuities at the edge of some spikes.

To interpret the observation in Fig. 2, we recall that the temporal characteristics of a SASE pulse are those of chaotic light due to the noisy startup. Under the one-dimensional, cold beam approximation with the electron energy chirp considered, the electric field in the exponential growth regime is a sum of $N_{\mathrm{e}}$ wave packets [11-13]

$$
E(t, z)=E_{o}(z) \sum_{j=i}^{N_{e}} \exp \left[i\left(\omega_{0}+\Phi^{\prime \prime}{ }_{\gamma}\left(t-t_{0}\right)\right)\left(t-t_{j}\right)+\frac{\left(t-t_{j}-z / v_{g}\right)^{2}}{4 \sigma_{t}^{2}}\left(1+\frac{i}{\sqrt{3}}\right)\right],
$$

where $N_{\mathrm{e}}$ is the total number of electrons in the bunch, $\Phi_{\gamma}^{\prime \prime}=\omega_{0} c \sigma_{\delta} / \sigma_{\mathrm{z}}$ with $\omega_{0}$ being instantaneous resonant frequency at $t_{0}, E_{0}(z)$ contains the exponential growth factor, $t_{\mathrm{j}}$ is the arrival time of the $j^{\text {th }}$ electron, $v_{\mathrm{g}}$ is the group velocity of each wave packet with the rms coherent 
length $\sigma_{\mathrm{t}} \approx\left(N_{u} / \rho\right)^{1 / 2} / 2 \omega_{\mathrm{r}}, N_{u}$ is the number of undulator periods, and $\rho$ is the FEL scaling parameter [2]. Clearly, an intrinsic chirp exists within a coherent spike as indicated by the quadratic phase term of $\left(t-t_{\mathrm{i}} \mathrm{-Z} / v_{\mathrm{g}}\right)^{2} / 4 \sqrt{3} \sigma_{\mathrm{t}}^{2}$ in Eq. (1), and an overall frequency chirp exists from spike to spike through $\Phi^{\prime \prime}{ }_{\gamma}$. Collecting all coefficients of quadratic terms in Eq. (1) and taking into account the dispersion in the collecting optics, the chirp observed by the FROG is

$$
\phi^{\prime \prime}=\frac{d^{2} \phi}{d t^{2}}=2 \operatorname{Im}(\Gamma)=2 \frac{\Omega \Theta+\Phi_{m}^{\prime \prime}}{\Omega^{2}+\Phi_{m}^{\prime \prime 2}},
$$

where $\Omega=4 \sigma_{\mathrm{t}}^{2}-\Phi^{\prime \prime}{ }_{\mathrm{m}} \Theta, \Theta=1 / 3^{1 / 2}+4 \sigma_{\mathrm{t}}^{2} \Phi^{\prime \prime}{ }_{\gamma}$, and $\Phi^{\prime \prime}{ }_{\mathrm{m}}$ is the dispersion in the collecting optics of 1.9-cm fused silica.

The experimentally measured chirp $\left|\phi^{\prime \prime}\right|$ is given in Fig. 2 as a function of the coherence length (approximated as the spike length) for individual spikes. Due to the intrinsic jitter and complicated behavior of each spike, the data are rather scattered. Even so, one can clearly identify a sharp dip at around $\sigma_{\mathrm{t}}=75 \mathrm{fs}$, which corresponds to $\Theta \approx 0$ in Eq. (2). By slightly adjusting the electron beam energy chirp in Eq. (2), we are able to fit the position of the dip at about $-28 / \mathrm{m}$ (see curves in Fig. 2).

The results in Fig. 2 unambiguously reveal the positive intrinsic SASE chirp. They also verify that the electron beam energy chirp directly maps into the FEL output, a key process for compressing and slicing the pulse from future X-ray FELs. Figure 2 also serves as an independent measurement of the electron bunch energy chirp.

The FROG traces also provide rich information on the statistics of the SASE output, especially the temporal information on these pulses. As the FROG traces provide the information in the frequency domain simultaneously, a correlation analysis between the time and the frequency domain is now possible. In Table 2 we give a summary of the important properties 
such as the coherent length, spike numbers, and spike separation in both the time and frequency domains. The numbers are in basic agreement with the statistical analysis $[11,12]$, which shows that for an electron bunch with an FWHM duration $T$, the SASE pulse is organized into $M \approx T / 4 \sigma_{\mathrm{t}}$ coherent spikes separated roughly by $4 \sigma_{\mathrm{t}}[12]$, and the SASE intensity fluctuates from shot to shot with a relative fluctuation $1 / \mathrm{M}^{1 / 2}$. A more detailed statistic analysis is underway.

In conclusion, we observed a positive intrinsic chirp in the SASE FEL spikes, and we confirmed that the energy chirp in the electron bunch does map to the SASE output. These observations have very important applications for future X-ray FEL sources in pulse engineering and manipulation. We also give the statistics on the time- and frequency-domain properties of the FEL.

The authors are grateful for help by O. Markarov and R. Dejus for performing the gain length measurement and processing the data. We also thank K.-J. Kim and L. Teng for insightful discussions. This work is supported by the U. S. Department of Energy, Office of Basic Energy Sciences, under Contract No. W-31-109-ENG-38. 


\section{References}

[1] A. M. Kondratenko, E. L. Saldin, Sov. Phys. Dokl. 24 (12), 986 (1979).

[2] R. Bonifacio, C. Pellegrini, L. M. Narducci, Opt. Commun. 50, 373 (1984).

[3] M. Cornacchia et al., "Linac Coherent Light Source (LCLS) Design Study Report," Report SLAC-R-521 (Stanford Linear Accelerator Center, Stanford, CA), revised 1998.

[4] R. Brinkmann, G. Materlik, J. Rossbach, A. Wagner, Eds., "Conceptual Design of a 500 $\mathrm{GeV} \mathrm{e}^{+} \mathrm{e}$ Linear Collider with Integrated X-Ray Laser Facility,” DESY Report DESY97048 (Deutsches Elektronen-Synchrotron, Hamburg), 1997.

[5] S. V. Milton et al., Science 292, 1953 (2001).

[6] V. Ayvazyan et al., Phys. Rev. Lett. 88, 104802-1 (2002).

[7] A. Tremaine et al., Phys. Rev. Lett. 88, 204801-1 (2002).

[8] S. G. Biedron et al., Nucl. Instrum. Methods Phys. Res. A 483, 94 (2002).

[9] R. Trebino et al., Rev. Sci. Instrum. 68, 3277 (1997).

[10] E. Gluskin et al., Nucl. Instrum. Methods Phys. Res. A 429, 358 (1999).

[11] R. Bonifacio et al., Phys. Rev. Lett. 74, 70 (1994).

[12] K.-J. Kim, "Towards X-ray free electron lasers," AIP Conference Proc 413, ed. R. Bonifacio and W. A. Barletta (American Insitute of Physics, New York), p. 3 (1997).

[13] C. B. Schroeder et al., Nucl. Instrum. Methods Phys. Res. A 483, 89 (2002). 
Table 1. Main experimental parameters

\begin{tabular}{ccc}
\hline \hline & Data set A & Data set B \\
Bunch charge & $1 \mathrm{nC}$ & $0.57 \mathrm{nC}$ \\
rms bunch length $\left(\sigma_{\mathrm{z}}\right)$ & $0.5 \mathrm{ps}$ & 0.3 \\
rms uncorrelated energy spread $\left(\sigma_{\delta}^{\mathrm{inc}}\right)$ & $0.7 \%$ & $0.6 \%$ \\
Bunch chirp $\left(\sigma_{\delta} / \sigma_{\mathrm{z}}\right)$ & -25 per meter & - \\
rms normalized emittance & $9 \pi \mathrm{mm} \mathrm{mrad} \quad 5 \pi \mathrm{mm} \mathrm{mrad}$ \\
Undulator period $\left(\lambda_{\mathrm{u}}\right)$ & $3.3 \mathrm{~cm}$ \\
Undulator peak field & $1 \mathrm{~T}$ \\
Undulator length $($ each $)$ & $2.4 \mathrm{~m}$ \\
Undulator strength parameter $(K)$ & 3.1 \\
Beam energy $\left(\gamma \mathrm{yc}^{2}\right)$ & $217 \mathrm{MeV}$ \\
Nominal radiation wavelength $(\lambda)$ & $530 \mathrm{~nm}$ \\
\hline \hline
\end{tabular}


Table 2. Measured parameters of the SASE FEL output

\begin{tabular}{|c|c|c|c|}
\hline Parameters & & Data set A & Data set B \\
\hline \multirow[t]{8}{*}{ General } & Gain length $L_{\mathrm{G}}(\mathrm{m})$ & $0.68 \pm 0.10$ & $0.77 \pm 0.07$ \\
\hline & Energy per pulse $W(\mu \mathrm{J})$ & 60 & - \\
\hline & Coherent modes ${ }^{a}$ & - & 1.2 \\
\hline & rms coherence length $\sigma_{\mathrm{t}}(\mathrm{fs})^{b}$ & $53 \pm 16$ & $56 \pm 10$ \\
\hline & Time bandwidth product & $0.52 \pm 0.31$ & $0.54 \pm 0.28$ \\
\hline & FWHM pulse duration (fs) & $197 \pm 105$ & $172 \pm 71$ \\
\hline & FWHM bandwidth (nm) & $2.6 \pm 0.8$ & $3.0 \pm 0.9$ \\
\hline & rms bandwidth (rad/s) & $(7.3 \pm 2.4) \times 10^{12}$ & $(8.5 \pm 2.5) \times 10^{12}$ \\
\hline \multirow[t]{5}{*}{ Spikes: frequency domain } & Number of spikes ${ }^{c}$ & $2.0 \pm 1.0$ & $1.3 \pm 0.5$ \\
\hline & Spike separation $\Delta \lambda(\mathrm{nm})^{c}$ & $3.01 \pm 1.09$ & $3.1 \pm 0.9$ \\
\hline & $\Delta \omega(\mathrm{rad} / \mathrm{s})$ & $(20 \pm 6) \times 10^{12}$ & $(21 \pm 5) \times 10^{12}$ \\
\hline & rms spike width $(\mathrm{nm})^{d}$ & $0.89 \pm 0.34$ & $1.2 \pm 0.3$ \\
\hline & $(\mathrm{rad} / \mathrm{s})$ & $(5.9 \pm 2.3) \times 10^{12}$ & $(7.8 \pm 1.9) \times 10^{12}$ \\
\hline \multirow[t]{3}{*}{ Spikes in time domain } & Number of spikes ${ }^{c}$ & $2.0 \pm 1.0$ & $1.3 \pm 0.5$ \\
\hline & Separation $\Delta t(\mathrm{fs})^{c}$ & $175 \pm 64$ & $206 \pm 61$ \\
\hline & Spike length (fs) ${ }^{d}$ & $53 \pm 16$ & $56 \pm 10$ \\
\hline
\end{tabular}

a. $\quad$ Measured from energy fluctuation

b. Assumed to be similar to spike length, measure only for well separated spikes

c. $\quad$ Using 0.05 intensity threshold

d. $\quad$ For well separated spikes only 
Figure captions

Figure 1. Example FROG traces and their retrievals showing instantaneous frequency (black), phase (blue), and intensity (red) as a function of time. (a) A single-spike dominated case and (b) a multiple-spike case. Note that both raw traces show a symmetric pattern in the time direction with unbalanced intensity. The sharp spikes in the frequency plots are due to the phase discontinuity. The retrievals are shown in the correct time direction.

Figure 2. Magnitude of the chirp of the SASE spikes. The data points are experimental measurement using the FROG. The curves are fit using Eq. (2) with an electron beam energy chirp of $-24 / \mathrm{m}$ (dashed line), -28/m (solid line), and -33/m (dotted line). 


\section{(a) Shot A0070}

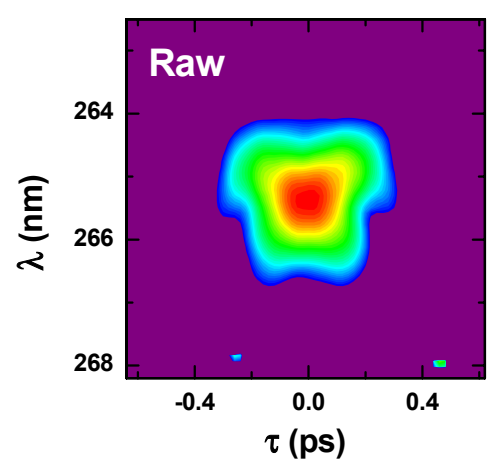

(b) Shot A0442

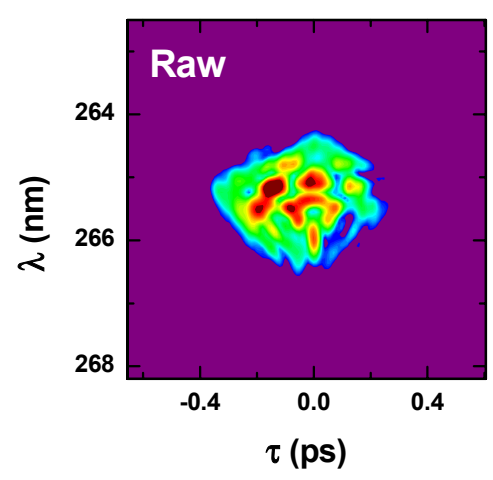

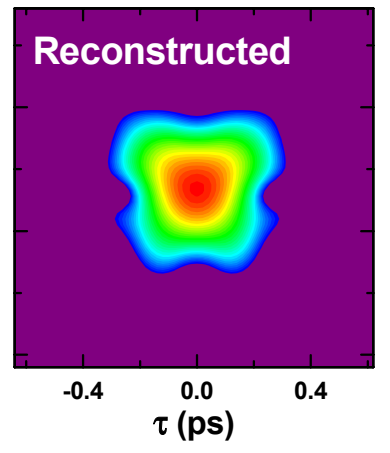
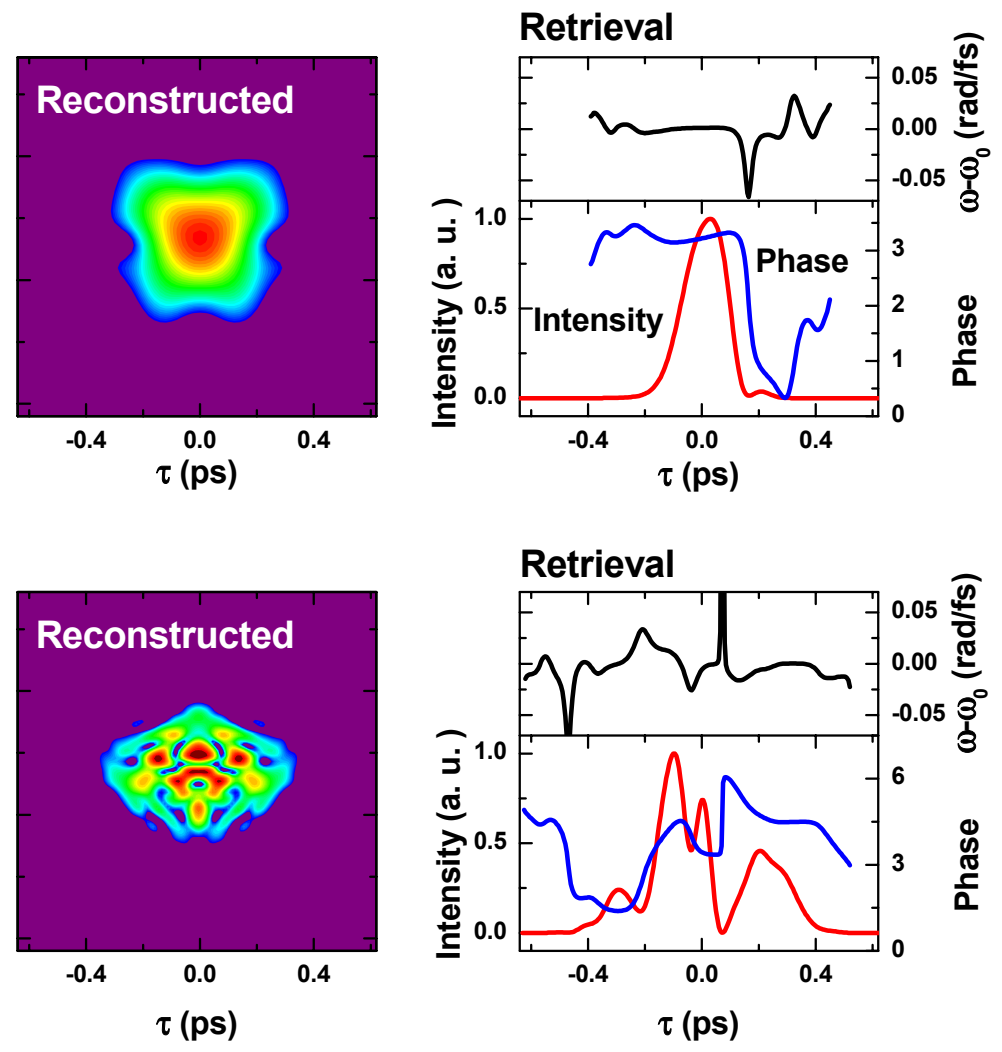

Figure 1. Example FROG traces and their retrievals showing instantaneous frequency (black), phase (blue), and intensity (red) as a function of time. (a) A single-spike dominated case and (b) a multiple-spike case. Note that both raw traces show a symmetric pattern in the time direction with unbalanced intensity. The sharp spikes in the frequency plots are due to the phase discontinuity. The retrievals are shown in the correct time direction. 


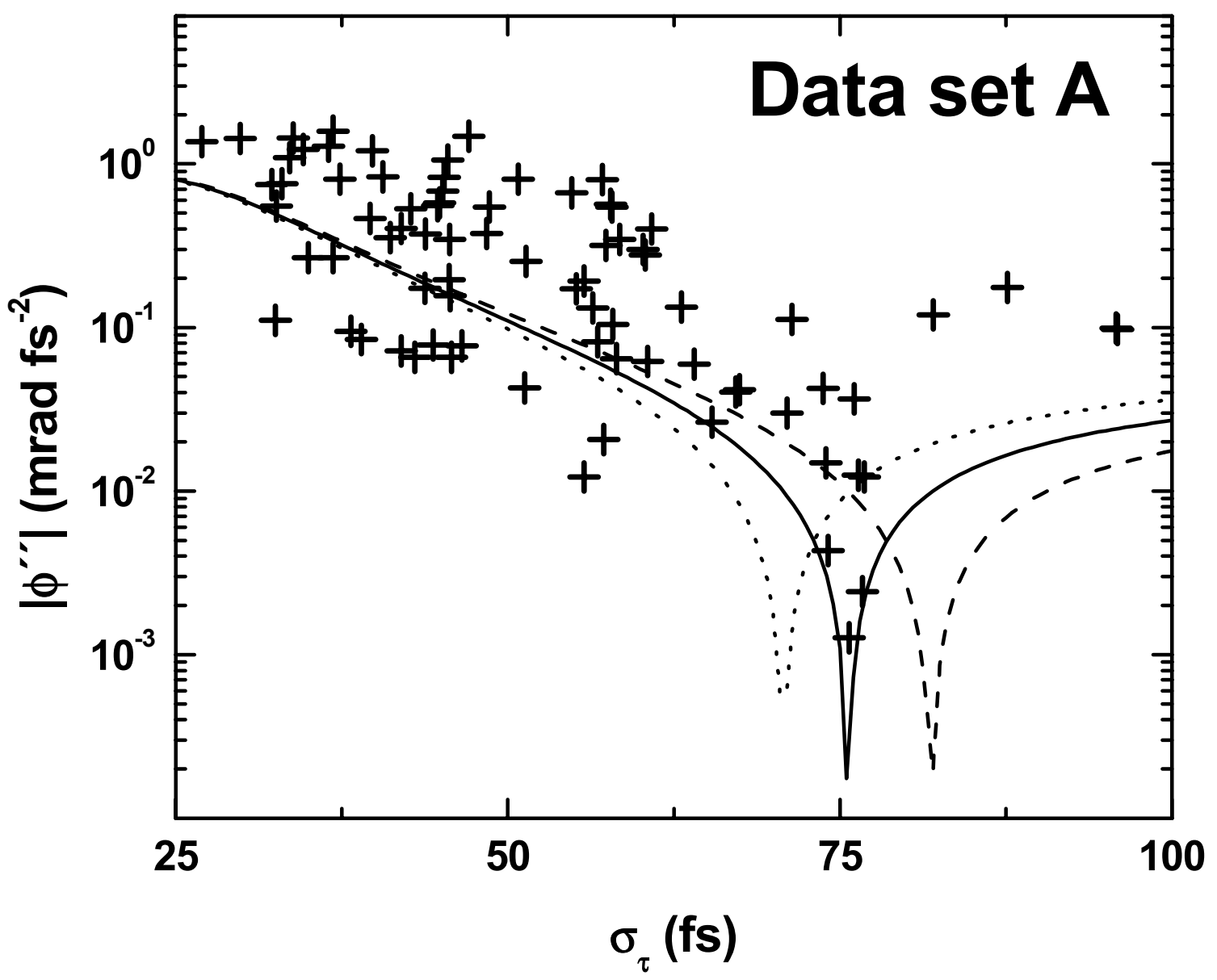

Figure 2. Magnitude of the chirp of the SASE spikes. The data points are experimental measurement using the FROG. The curves are fit using Eq. (2) with an electron beam energy chirp of -24/m (dashed line), $-28 / \mathrm{m}$ (solid line), and -33/m (dotted line). 